\title{
Electrically Controlled Delivery of Cargo into Single Human Neural Stem Cell
}

\author{
Tae-Hyung Kim, ${ }^{\dagger \dagger}$ Hyeon-Yeol Cho, ${ }^{\dagger}$ Ki-Bum Lee, ${ }^{\ddagger}$ Seung U. Kim, ${ }^{\S, \|}$ and Jeong-Woo Choi ${ }^{*}{ }^{\dagger}$ \\ ${ }^{\dagger}$ Department of Chemical \& Biomolecular Engineering, Sogang University, 35 Baekbeom-ro, Mapo-gu, Seoul 121-742, Korea \\ ${ }^{\star}$ Department of Chemistry and Chemical Biology, Rutgers, The State University of New Jersey, 610 Taylor Road, Piscataway, New \\ Jersey 08854, United States \\ ${ }^{\S}$ Medical Research Institute, Chungang University College of Medicine, 221 Heukseok-dong, Dongjak-gu, Seoul 156-756, Korea \\ "Department of Medicine, University of British Columbia, 2329 West Mall, Vancouver, British Columbia V6T 1Z4, Canada
}

\section{Supporting Information}

ABSTRACT: Nanoprobe-based techniques have emerged as an efficient tool for the manipulation and analysis of single cells. Here, we report a powerful whole-electrical single-cell manipulation tool that enables rapid and controllable delivery of cargo into single neural stem cells with precision monitoring of the cell penetration process using a conductive nanoprobe. The highly electrically sensitive nanoprobes that were fabricated and the indium tin oxide electrode-integrated cell chip were found to be very effective for monitoring the cell penetration process via

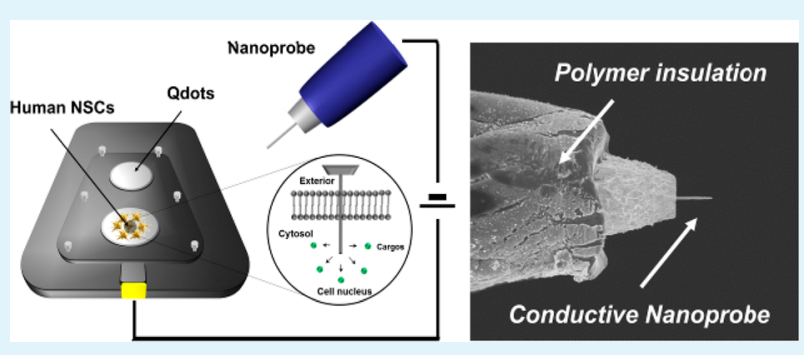
current changes that appear as spike-like negative currents. Moreover, the assembly of cargoes onto the nanoprobes was controllable and could reach its maximum load in a very short period of time $(<10 \mathrm{~min})$ based on the same electrical system that was used for monitoring cell penetration and without the need for any complex chemical linkers or mediators. Even more remarkably, the cargo assembled on the surface of the nanoprobe was successfully released in a very short period of time $(<10 \mathrm{~s})$, regardless of the surrounding intracellular or extracellular environments. The monitoring of cell penetration, assembly of quantum dots (QDs), and release of QDs into the intracellular environment were all accomplished using our whole-electrical system that combined a conductive nanoprobe with cell chip technology. This is a novel technology, which can eliminate complex and time-consuming steps owing to chemical modifications, as well as reduce the time needed for the delivery of cargo into the cell cytosol/nucleus during cell penetration, which is very important for reducing cell damage.

KEYWORDS: single-cell manipulation, electrical assembly and release, nanoprobe, quantum dots, human neural stem cell

\section{INTRODUCTION}

Due to the fact that cells are considered the basic "building block" of whole living organisms, single-cell analysis is incredibly important to obtain a variety of invaluable information, which would otherwise be impossible to acquire from large populations of cells or complex tissues. ${ }^{1}$ Thanks to the rapid development of nanotechnology, nanoprobe-based single-cell manipulation tools have emerged as an efficient tool for the manipulation and analysis of single cells. ${ }^{2-5}$ Miyake and co-workers first reported a novel single-cell manipulation technique that utilized focused ion beam (FIB)-etched silicon tips and atomic force microscopy (AFM) to control and monitor the cell penetration process by the force applied to AFM tips for stabbing. ${ }^{6-8}$ The research was further extended to deliver fluorescein-tagged materials or green fluorescent protein (GFP)-encoded plasmid vectors for the manipulation of single cells. ${ }^{9,10}$ In a similar way, Bertozzi and co-workers reported an AFM-based single-,cell manipulation system using carbon nanotube (CNT)-conjugated AFM tips that successfully delivered quantum dots into the cell cytosol while minimizing cell membrane damage due to the small diameter of the $\mathrm{CNT}^{11}$ All of these reports utilizing silicon tips and AFM techniques were found to be effective for delivering materials of interest into the desired location within single cells with precise control over stabbing. However, complex chemical modifications steps were essential for both the materials and surfaces of the nanoprobes to attach and release the cargo into the cell cytosol or nucleus that were time-consuming and, more importantly, non-reusable. Moreover, since the delivery mechanisms used in these studies was based on disulfide breakage of chemical linkers that connected the cargo to the nanoprobes, the release of cargo was fully dependent on the intracellular environment and thus not controllable over long penetration times $(10-15 \mathrm{~min})$, which has negative effects on cell viability. To this end, Friedman and co-workers recently reported a CNT-based "cellular endoscope" for the delivery of

Received: July 10, 2014

Accepted: September 10, 2014

Published: September 10, 2014 
fluorescent materials into the cell cytosol by flowing fluid into single cells through the inner space of CNTs, which was rapid, controllable, and also effective for surface-enhanced Raman scattering (SERS)/electrochemical study. ${ }^{12}$ While it is obvious that this new single-cell manipulation/analysis system is excellent for controlling the delivery of materials of interest into target cells, the fabrication steps required for this CNTbased cellular endoscope are relatively complex, making it hard for general single-cell study.

We have previously reported a new electrical method which was very effective for monitoring changes in cell viability/ proliferation, which corresponded to variations that occurred in the extracellular environment such as the extracellular matrix (ECM) protein immobilized on the electrode surface, the peptide or metal nanopatterned surface, the addition of anticancer drugs, and environmental toxins. ${ }^{13-17}$ Recently, this technology was further utilized to confirm the preference of human neural stem cells (NSCs) for nanopatterned surfaces in terms of cell adhesion and proliferation, as well as to validate the negative effects of doxorubicin on human NSCs (hNSCs). ${ }^{18}$ From these endeavors, we successfully developed a new concept, methodology, and technology that integrated electrical/electrochemical techniques with various types of living cells. Hence, we hypothesized that these electrical techniques, when integrated into cell chip technology, could be useful for not only the detection of cell viability but also single-cell manipulation, thereby enabling the rapid, simple, and controlled delivery of materials of interest into target cells.

In the present study, we report a powerful whole-electrical single-cell manipulation tool that enables rapid and controllable delivery of cargo into single NSCs while monitoring the cell penetration process using a conductive nanoprobe. A polymerinsulated nanoprobe was fabricated by two-step electrochemical and FIB etching to penetrate the cell membrane without potentially damaging target cells. Electrical monitoring of cell penetration was attempted by applying weak voltage between the cells cultured on the indium tin oxide (ITO) electrodeintegrated cell chip and the polymer-insulated conductive nanoprobe. The electrical force was further applied between the nanoprobe and cell chip to assemble quantum dots (QDs) on the surface of the nanoprobe and to detach it by applying the voltage opposite to the one used for the assembly while the fluorescence intensities around the nanoprobe were monitored by the fluorescence microscopy simultaneously. After the confirmation of electrically driven assembly and release of QDs, the nanoprobe carrying concentrated negatively charged QDs was finally utilized to deliver QDs into the intracellular environment by applying the voltage opposite to the one used for the assembly.

\section{EXPERIMENTAL SECTION}

2.1. Materials. $\mathrm{Au}, \mathrm{Ag}, \mathrm{Pt}, \mathrm{Pt} / \mathrm{Ir}$ alloys (80:20), and Ir wires were purchased from Surepure Chemetals (Florham Park, NJ, USA). ITOcoated cover glass (thickness: $0.19 \mathrm{~mm}$ ) was obtained from SPI Supplies (West Chester, PA, USA). Calcium chloride and potassium hydroxide were purchased from Sigma-Aldrich (St. Louis, USA). The phosphate-buffered saline (PBS; pH 7.4, $10 \mathrm{mM}$ ) solution consisting of $136.7 \mathrm{mM} \mathrm{NaCl}, 2.7 \mathrm{mM} \mathrm{KCl}, 9.7 \mathrm{mM} \mathrm{Na}_{2} \mathrm{HPO}_{4}$, and $1.5 \mathrm{mM}$ $\mathrm{KH}_{2} \mathrm{PO}_{4}$ was purchased from Sigma-Aldrich. Dulbecco's modified Eagle's medium (DMEM) and antibiotics were purchased from Invitrogen (Carlsbad, CA, USA). Other chemicals used in this study were obtained commercially and were reagent grade.

2.2. Cell Culture. A clonal human neural stem cell (HB1.F3) originated from human telencephalon cells that had been immortalized by an amphotropic replication-incompetent retroviral vector encoding v-myc was utilized in this study. Details on the generation and characterization of HB1.F3 (F3) immortalized human NSC line was reported previously. ${ }^{19,20}$ F3 NSCs were cultured in DMEM supplemented with $10 \%$ fetal bovine serum (FBS), $100 \mathrm{U} / \mathrm{mL}$ penicillin and $100 \mu \mathrm{g} / \mathrm{mL}$ streptomycin. Cells were maintained under common cell culture conditions at $37^{\circ} \mathrm{C}$ in an atmosphere of $5 \% \mathrm{CO}_{2}$.

2.3. Cell Chip for Single-Cell Manipulation. Cell chip that can be inserted into the microscope incubator were designed by our group and fabricated by Live Cell Instrument (Seoul, Korea). Chip was designed to have space for the thin coverslip which can be automatically connected to the gold electrode when it assembled completely. Two wells separated from each other were used for both cell culture and electrical assembly of QDs on the nanoprobe.

2.4. Fabrication of Polymer-Insulated Nanoprobe. To fabricate sharp tips that are proper for cell penetration, $\mathrm{Au}, \mathrm{Pt}$, and $\mathrm{Pt} / \mathrm{Ir}$ alloy wire with diameter of $0.25 \mathrm{~mm}$ were used. Twenty percent saturated $\mathrm{CaCl}_{2}$ solution and cylinder-type carbon electrode were used as an electrolyte and counter electrode for electrochemical etching, respectively. Direct current (dc;50 V) was applied to Au wire and carbon counter electrode until the currents reach zero. The concentrations of $\mathrm{CaCl}_{2}$ were increased to enlarge the average diameter of electrochemically etched electrode. In the case of Pt or Pt/ Ir alloys, alternating current (ac) voltages were applied by varying its peak-to-peak value and frequencies to fabricated differently sized metal electrode. The electrochemically etched probes were then coated with parylene C (500 $\mathrm{nm}$ to $2 \mu \mathrm{m})$ for the insulation prior to the second etching by FIB (SMI3050SE, The Seiko Instruments or NOVA 200, FEI Company. In the case of tungsten nanoprobe, micrometer-sized tungsten microelectrode protected by polymer insulation was purchased from FHC (Bowdoin, ME, USA) and directly applied to FIB second etching.

2.5. Electrical Monitoring of Cell Penetration. Polymerinsulated nanoprobe was first inserted into the electrode holder that is connected to a cell manipulator (Patchman NP 2, Eppendorf) which enables precise control of the movement of the nanoprobe for cell penetration. At the same time, the electrode holder was connected to an electrical source meter (Model 2601A, Keithley) for electrical monitoring of cell penetration. The nanoprobe and ITO electrode were set as negative and positive electrodes, respectively. DC $(20 \mathrm{nV})$ was applied to monitor the changes of electrical current induced by cell penetration while the number of power line cycles (NPLC) was set to 20 to remove noises, as well as to increase the sensitivity of the system. For cell penetration, the nanoprobe was first approached to the target cell by controlling a cell manipulator while monitoring the movement of the nanoprobe by an optical microscope (Ti-U, Nikon). Once the nanoprobe reached the cell membrane, the movement of the nanoprobe was slowed using "ultrafine" mode of the cell manipulator to penetrate the cell membrane with minimal cell damages while maintaining an angle of $45^{\circ}$. The movement of the nanoprobe was completely stopped when a spikelike current appeared on $I-t$ graph that was monitored by an $I-V$ source meter. The detection time for the $I-t$ graph was $0.1-0.6 \mathrm{~s}$.

2.6. Electrical Assembly and Release of QDs. First, QDs were made water-soluble by capping thioglycolic acid (TA) as previously described. $^{15,21}$ Briefly, $100 \mu \mathrm{L}$ of QDs in toluene solution were transferred to $100 \mu \mathrm{L}$ of chloroform. After the addition of $1 \mathrm{~mL}$ of TA, the mixture was shaken vigorously for $2 \mathrm{~h}$, followed by washing it three times with acetone to remove free TA ligand. The solution containing water-soluble QDs $(100 \mu \mathrm{g} / \mathrm{mL})$ were then transferred to a cell chip for electrical assembly. The nanoprobe was immersed into deionized (DI) water containing QDs, followed by applying electrical force (1.5 V) between the nanoprobe and ITO electrode while the fluorescence of QDs around the nanoprobe was monitored by the fluorescence microscope simultaneously. Time-dependent images were recorded every 2 min using microscopic imaging software (NIS Element-basic, Nikon) to confirm the correlations between the time applied and the amount of QDs assembled on the surface of the nanoprobe. Since QDs are functionalized with TA having a negative charge, the 

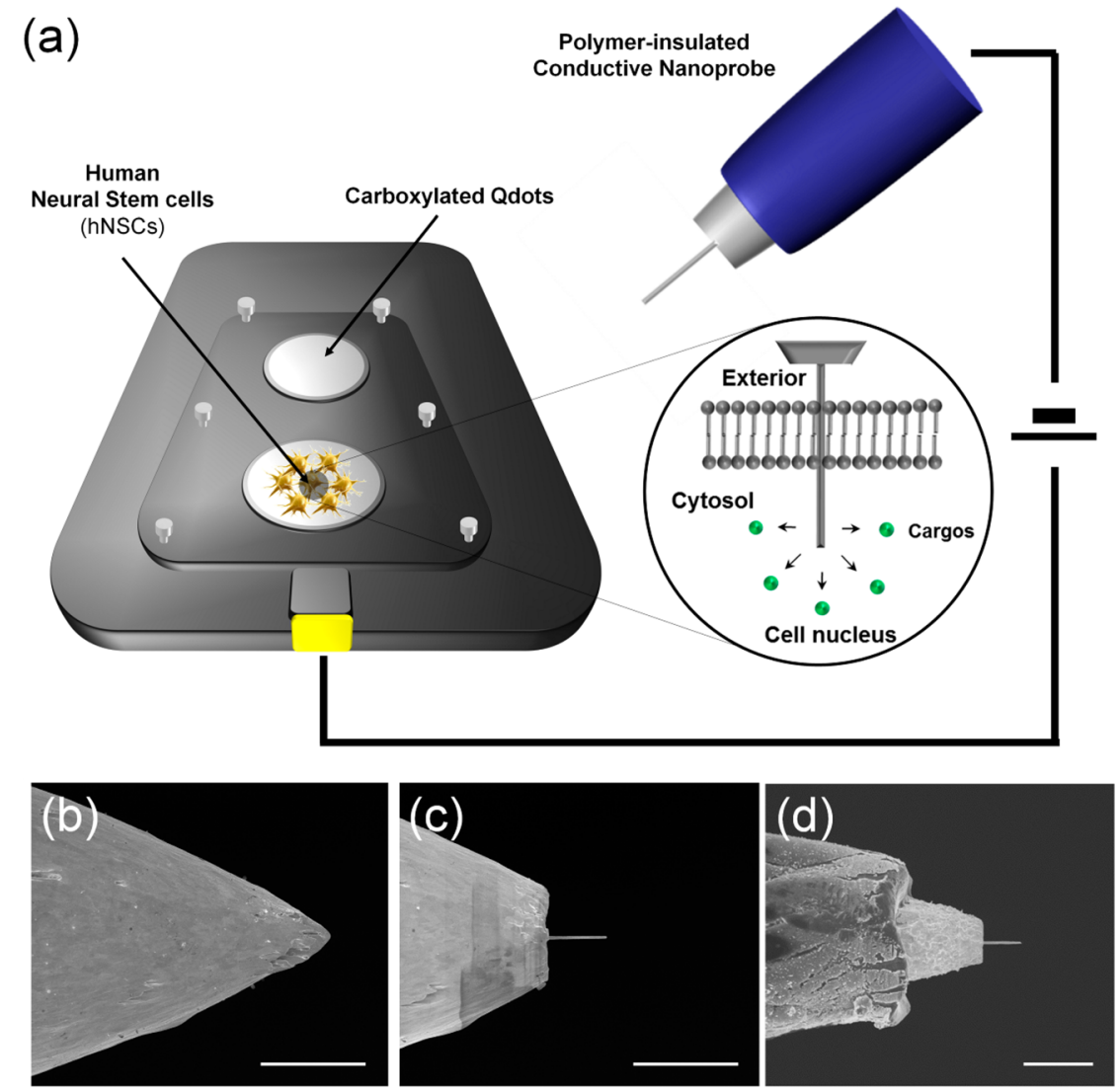

Figure 1. (a) Schematic diagram representing the whole setup used for single-cell manipulation. The ITO cover glass inserted-cell chip chamber containing two different wells were filled with QD-containing deionized water (DIW) and cell culture medium where hNSCs were grown on the ITO surface, respectively. The nanoprobe first immersed in the solution containing QDs for its assembly and moved to the well containing hNSCs for releasing QDs inside the cell membrane. Pt/Ir alloy electrode sharpened by (b) electrochemical etching and (c) 2nd FIB etching. (d) Parylene Cinsulated $\mathrm{Pt} / \mathrm{Ir}$ nanoprobe used for cell penetration. Scale bar $=10 \mu \mathrm{m}$.

nanoprobe and ITO electrode were set as positive and negative electrode, respectively.

The nanoprobe carrying QDs was then slowly moved to the well containing PBS ( $\mathrm{pH} 7.4$ ) or cell culture medium to monitor an electrical release of QDs. Once it reached the position that is close to the bottom of the ITO electrode (approximately $100 \mu \mathrm{m}$ above the ITO electrode), the voltage opposite to the one used for the assembly of QDs $(-1.5 \mathrm{~V})$ was applied to detach QDs assembled on the surface of the nanoprobe. Time-dependent images were recorded every $10 \mathrm{~s}$ using fluorescence microscope to confirm the amount of QDs detached. In the case of delivery of QDs into the intracellular environment of hNSCs, the voltage applied to detach QDs was reduced to $-1.0 \mathrm{~V}$ to prevent possible cell damage. Since the penetration is extremely hard to monitor using an optical microscope due to its small size, releasing QDs from the nanoprobe was conducted when spikelike negative current appeared on an $I-t$ graph.

\section{RESULTS AND DISCUSSION}

3.1. Fabrication of Polymer-Insulated Conductive Nanoprobe. The schematic diagram illustrates the entire experimental setup and the strategy for delivering QDs into single human NSCs with precisely controlled cell penetration that is also monitored by electrical current (Figure 1a, Figure S1a,b in the Supporting Information). The chip designed by our group has two wells that are capable of culturing cells on the ITO-deposited cover glass (Figure S1c). Once the ITO cover glass is assembled into the cell chip, the gold electrodes automatically touch the ITO surface, thereby allowing for the flow of electrons. In the case of this study, only one well was used for cell culture while the other well was used for electrical assembly of QDs on the conductive nanoprobe surface. Specifically, the nanoprobes used in this study were first sharpened using an electrochemical etching method. Gold, silver, platinum, platinum/iridium alloys, and tungsten are all possible candidates that can be used to reduce the diameter to less than $1 \mu \mathrm{m}$ by changing the composition of the electrolytes, the intensity of voltages, and its patterns $(\mathrm{dc} / \mathrm{ac})$. Figure $1 \mathrm{~b}$ and Figure $\mathrm{S} 2 \mathrm{a}-\mathrm{c}$ show $\mathrm{Pt} / \mathrm{Ir}$ alloy and gold wires sharpened by electrochemical etching method.

These wires having diameter of $100 \mathrm{~nm}$ to $2 \mu \mathrm{m}$ were further etched using a focused ion beam (FIB) to fabricate a rodlike structure with a diameter of $250 \mathrm{~nm}$ to $1 \mu \mathrm{m}$ that is proper for the cell penetration studies as shown in Figure $1 \mathrm{c}(\mathrm{Pt} / \mathrm{Ir}$ alloy, Pt:Ir = 80:20) and Figure S2d-f $(\mathrm{Au}){ }^{6,8}$ However, these nanoprobes were not sensitive enough to monitor the cell penetration process due to the huge conducting surface of the nanoprobe, which can act as an electron acceptor when it is immersed into a solution of electrolytes. Hence, the nanoprobe was first coated with parylene $C$ to insulate the entire surface and finally etched by FIB to make the nanoprobe conductive only at the end of the tip. Figure $1 \mathrm{~d}$ shows a $\mathrm{Pt} / \mathrm{Ir}$ alloy nanoprobe insulated with the parylene $C$ layer and sharpened by FIB, which is designed for monitoring cell penetration using the electrical method.

3.2. Electrical Monitoring of Cell Penetration Using Nanoprobe and Cell Chip. For electrical monitoring of cell 

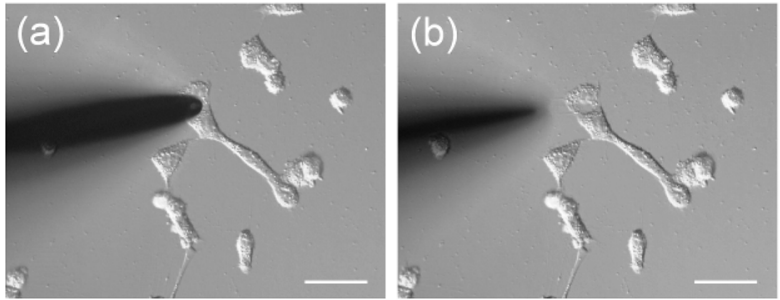

(e)

Cell touch No penetration Removal
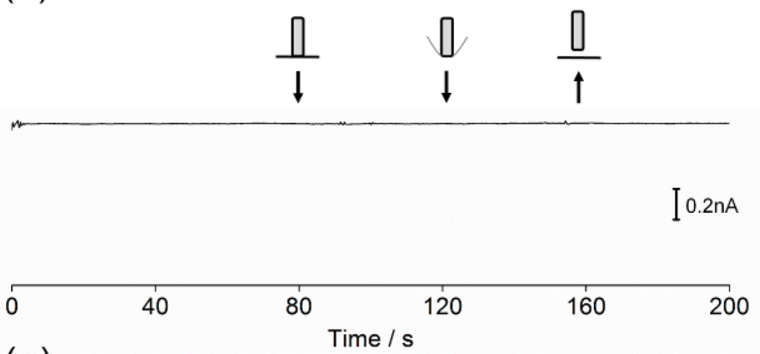

(g)

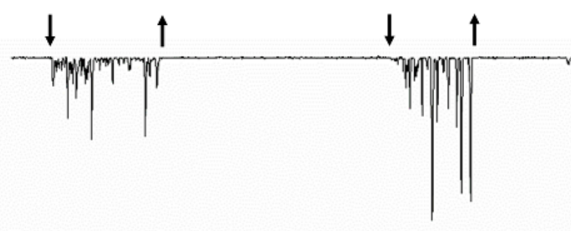

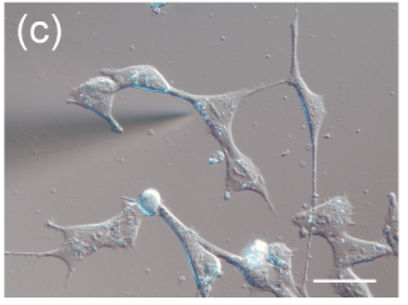

(f)

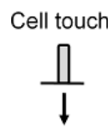

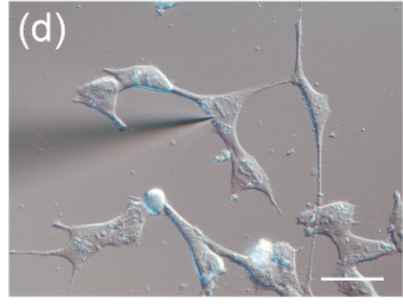

Penetration

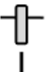

100

200

300

400

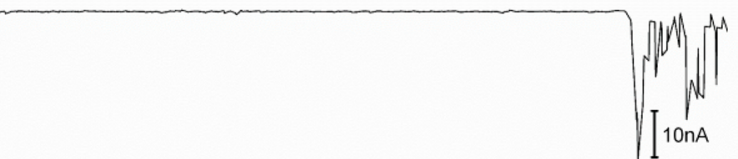

\begin{tabular}{lllllllllll}
\hline 0 & 10 & 20 & 30 & 40 & 50 & 60 & 70 & 80 & 90 & 100
\end{tabular}

Time/s

Figure 2. Electrical monitoring of cell penetration using a nanoprobe. DIC images of hNSCs (a) during the cell membrane touch using a dull tip, (b) after the removal of the dull tip, and (c) before and (d) after the cell penetration using a nanoprobe. (e) Current responses during cell touch using a dull tip which is not capable of penetrating the cell membrane. (f) Current signals during the cell penetration using a nanoprobe. ( $g$ ) Monitoring of multiple cell penetration for single hNSC using a nanoprobe. Electrolyte used for the study was normal DMEM medium containing 10\% FBS and $1 \%$ antibiotics. Scale bar $=50 \mu \mathrm{m}$.

penetration, many different types of metal nanoprobe (e.g., gold, platinum, Pt/Ir alloy, and tungsten) were applied, but only $\mathrm{Pt} / \mathrm{Ir}$ and tungsten nanoprobe were mechanically stable for multiple penetration of single cells due to its high shear modulus. $^{22}$ Hence, $\mathrm{Pt} / \mathrm{Ir}$ alloy or tungsten nanoprobe fully covered with parylene $\mathrm{C}$ polymer except for the area etched by FIB was finally used as a cell penetrating material (Figure S3a), which also showed excellent electrical characteristics that is critical for the sensitive monitoring of cell penetration (Figure $\mathrm{S} 3 \mathrm{~b})$. The final nanoprobe had a diameter of $250 \mathrm{~nm}$ with a height of $5 \mu \mathrm{m}$, resulting in $7.02 \times 10^{-6} \mathrm{~mm}^{2}$ of total conducting area.

As shown in Figure 2e, no changes of electrical currents were detected while touching single hNSC on ITO-coated coverslip using electrochemically etched normal dull tips (Figure 2a,b). However, huge spikelike negative currents appeared in the $I-t$ graph when cells were penetrated by the polymer-insulated FIB-sharpened nanoprobe with the application of $20 \mathrm{nV}$ (Figure 2c,d and Figure 2f). This phenomenon may be due to the cell membrane potential which is normally negatively charged with respect to the external cellular environment. It is well-known that cells keep its negative potential $(-40$ to -80 $\mathrm{mV}$ ) by controlling different types of ion channels (e.g., potassium, sodium, and chloride ion channel), which could be a useful source for operating many different kinds of membraneembedded "molecular devices". ${ }^{23-25}$ However, in this study, this cell membrane-mediated difference of electric potential, which can be considered as one kind of biological battery, will be ruptured by the insertion of the nanoprobe, thereby inducing bursting of electrons between the outer and inner membrane. Since the nanoprobe is electrically sensitive and is composing a complete electrical system with ITO bottom electrode, bursting of electrons induced by the insertion of the nanoprobe could be detected sensitively which might be responsible for the spikelike currents appearing on the $I-t$ graph. This hypothesis can be partially supported by previously reported studies utilizing a phi29 RNA connector-embedded artificial membrane that was highly effective for the ultrasensitive sensing of material of interest, ${ }^{26-29}$ which was even possible to discriminate double- and single-stranded DNA, ${ }^{28}$ as well as the real-time detection of single colon cancer specific antibody in serum. ${ }^{27}$ In this paper, authors claimed that materials of interest can be detected by applying voltage in two different environments separated by artificial membrane (insulator) which sensitizes phi29 RNA connector (probe) to the target analytes, resulting in the changes of currents which is similar to our model. However, it is true that additional confirmations should be followed to reveal the exact mechanism of current changes due to the cell penetration using the nanoprobe. To confirm reproducibility, repetitive cell penetrations were conducted on single human NSC without changing the nanoprobe used for cell stabbing. Remarkably, the spikelike negative currents continuously appeared in the $I-t$ graph whenever the nanoprobe penetrated the cell membrane, which was effective for sensitive monitoring of cell penetration fully based on the electrical method (Figure $2 \mathrm{~g}$ ). The 
nanoprobe also can be selectively inserted into cell cytosol or the nucleus by precisely monitoring the position of the nanoprobe using the optical microscope with a highmagnification lens $(100 \times)$ that enables clear distinguishment of the cell nucleus and cytosol (Figure S4). Hence, it can be concluded that the fabricated polymer-insulated nanoprobe combined with cell chip technology could be highly useful for single-cell manipulation, especially for the confirmation of cell penetration which is hard to detect using a conventional optical microscope.

3.3. Controlled Electrical Assembly of Quantum Dots on the Surface of the Nanoprobe. After the successful monitoring of cell penetration using the polymer-insulated nanoprobe, the electrical assembly of negatively charged QDs was attempted. To this end, the nanoprobe was first immersed in DI water containing carboxylated QDs, followed by the application of voltage between the nanoprobe and the ITO surface to induce the accumulation of QDs on the surface of the nanoprobe. Carboxyl groups were intentionally functionalized on the surface of QDs using thioglycolic acid to make it watersoluble, as well as to give negative charge on its surface which is essential for electrically driven assembly of QDs. Since QDs are a strong fluorescence material with low photobleaching effects, the movement and assembly of QDs around the surface of the nanoprobe were easily monitored by an inverted fluorescence microscope, simultaneously (Figure 3a).

As shown in Figure 3b, QDs start to assemble on the surface of the nanoprobe when voltage $(1.5 \mathrm{~V})$ was applied. Interestingly, only $3 \mathrm{~min}$ was enough to reach $40 \%$ fluorescence intensity around the surface of the nanoprobe. The relative fluorescence intensity reached $60 \%$ when voltage was applied for $9 \mathrm{~min}$, indicating that assembly of QDs around the surface of the nanoprobe was decreased, corresponding to the increase of time, which might be contributed by the limited surface area of the nanoprobe attracting negatively charged QDs by electrical force (Figure 3c). No assembly of QDs on the surface of the nanoprobe was observed for the same period of time that proved concentrated QDs around the surface of the nanoprobe was fully driven by electrical force applied between the nanoprobe and ITO electrode (Figure $3 b, c$ ). This is quite remarkable since no chemical linkers are needed to attach cargoes on the nanoprobe that normally require a few days for successful surface functionalization.

3.4. Electrical Release of Quantum Dots from Nanoprobe. After the successful electrical assembly of QDs on the surface of the nanoprobe, the voltage opposite to the one used for the assembly of QDs was applied to the nanoprobe for releasing QDs from the surface, which was also monitored by a fluorescence microscope (Figure 4a). Remarkably, most of the QDs attached on the surface of the nanoprobe were successfully released within $20 \mathrm{~s}$, which was confirmed by the relative fluorescence intensities achieved from five different spots of the nanoprobe in a time-dependent manner. Moreover, only $40 \mathrm{~s}$ was sufficient to reach negligible intensity of fluorescence, indicating that all of the detectable QDs were detached from the surface of the nanoprobe.

The electrical assembly $(5 \mathrm{~min})$ and release process $(20 \mathrm{~s})$ was further repetitively conducted using the same nanoprobe to confirm the reproducibility and showed outstanding performance for $n=3$ times of assembly and release as shown in Figure S5a. Relative fluorescence values achieved from Figure S5a also showed stable increase and decrease of the intensities that corresponds to the voltage applied to the nanoprobe (Figure
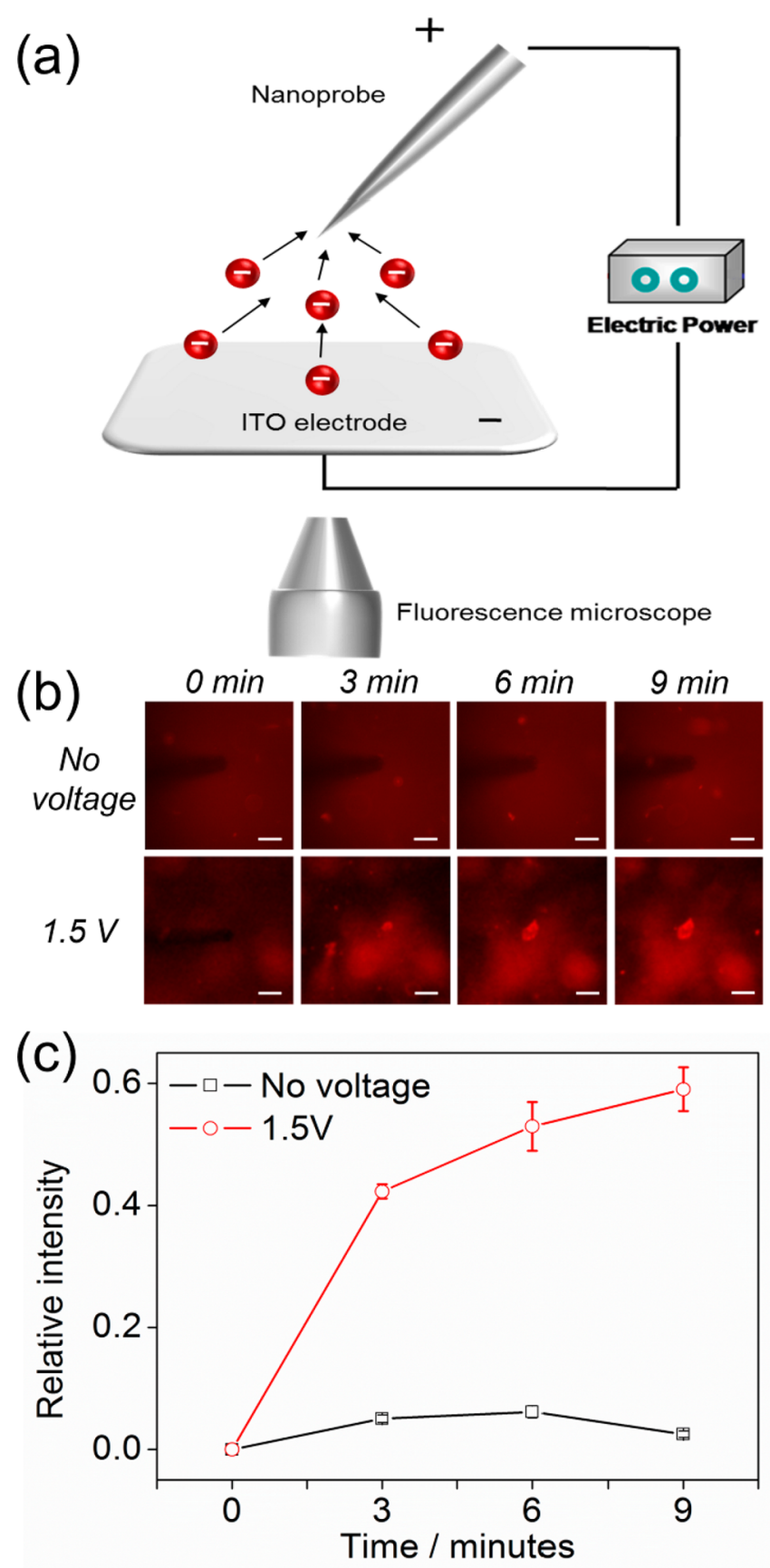

Figure 3. (a) Schematic diagram representing electrical assembly of QDs on the surface of a nanoprobe while $1.5 \mathrm{~V}$ voltage was applied between the ITO-coated cover glass and the nanoprobe. (b) Images of a nanoprobe immersed in QD-containing DIW with or without applying $1.5 \mathrm{~V}$ voltage which were monitored by an inverted fluorescence microscope. (c) Relative fluorescence intensities of QDs attached on the surface of a nanoprobe with different times. Relative fluorescence intensity was calculated by the values obtained from intensity profiles of fluorescence around the nanoprobe (five different points) divided by its maximum value (255) and further subtracted by the relative fluorescence value at time $=0$. Scale bar $=25$ $\mu \mathrm{m}$.

S5b). More remarkably, no release of QDs assembled on the surface of the nanoprobe was observed when no voltage was applied for $5 \mathrm{~min}$ each, proving the stability of electrical forcedriven cargo attachment. This is a significant advantage of electrically controlled assembly and release of QDs because 
(a)
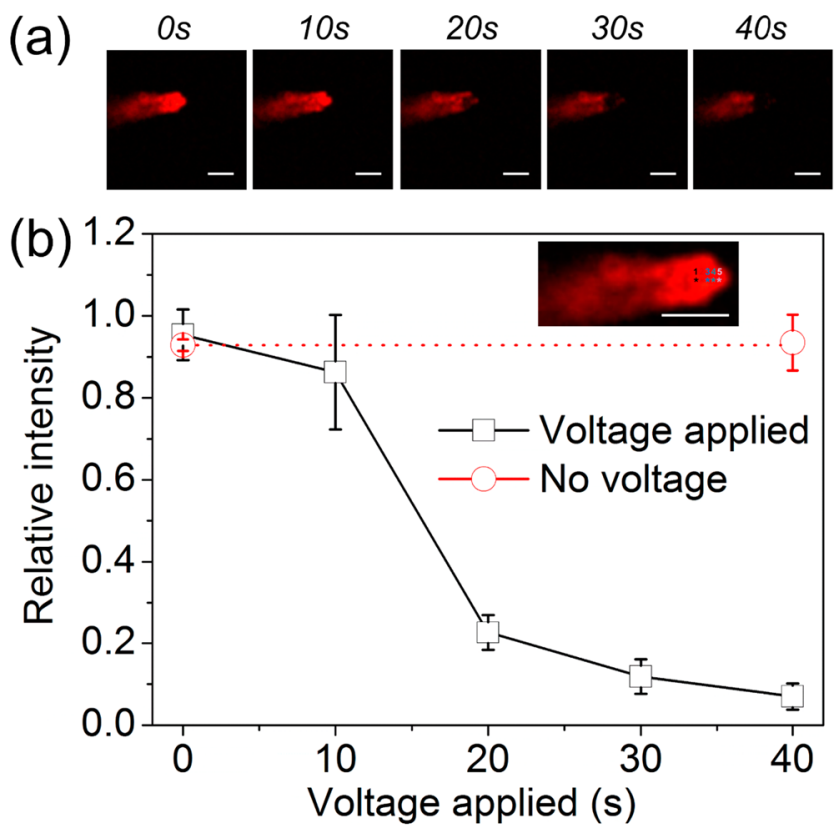

Figure 4. (a) Fluorescence images showing release of QDs on the nanoprobe by applying voltage. (b) Time-dependent relative fluorescence intensities of QDs assembled on the nanoprobe while reverse voltage was applied. Relative fluorescence values were calculated based on the fluorescence intensities averaged from the positions numbered as " 1 ", " 2 ", " 3 ", " 4 ", and " 5 " in the inset image of (b), divided by its maximum value (255). Scale bar $=25 \mu \mathrm{m}$.

molecules containing disulfide bonds that normally have been utilized to deliver materials of interest via the cleavage of the disulfide bond in the intracellular environment cannot be reused due to the free sulfide group remaining on the surface of the nanoprobe after the delivery of cargo into the cell.

3.5. Delivery of Cargo into Single Human Neural Stem Cell. After the confirmation of reproducibility of electrical assembly and release of cargo, the amount of QDs attached on the surface of the nanoprobe was adjusted by reducing the applied voltage and assembly time to avoid increasing the diameter of the nanoprobe, which may damage the cell membrane. As shown in Figure S6, both green- or red-emitting QDs were immobilized on the surface of the nanoprobe as a thin layer, which is different from that of QDs around the nanoprobe (Figure 3b, Figure 4a, Figure S5a). The nanoprobe carrying QDs on its surface were then moved to the other well in the cell chip to deliver the QDs into single hNSCs by changing the voltage from the positive to the negative direction. A very weak voltage $(20 \mathrm{nV})$ was applied before inducing the release of QDs on the surface of the nanoprobe to monitor the cell penetration process, which is very difficult to confirm by a microscope. The negative voltage $(-1.0 \mathrm{~V})$ was applied for only $10 \mathrm{~s}$ to detach the QDs from the nanoprobe, at which point a spikelike current appeared on the $I-t$ graph. This indicated that the nanoprobe penetrated the cell membrane.

As shown in parts (a) and (c) of Figure 5, the electrical forcedriven release of QDs from the surface of the nanoprobe could be monitored by DIC and fluorescence microscopy. The QDs detached from the surface of the polymer-insulated conductive nanoprobe were still remaining after washing with DPBS and cell culture medium, proving the successful delivery of QDs inside the cell membrane (Figure 5b,d). After the confirmation of delivery of red-emitting QDs into single hNSC, the same parameters used for the assembly and release of red-emitting QDs were used again to deliver green-emitting QDs into single hNSC. However, both the amount of QDs assembled on the surface of the nanoprobe and the QDs released into single hNSCs were found to be lower than that of red-emitting QDs, resulting in very weak intensity of QDs after the delivery (Figure S7). This may be due to the small size of greenemitting QDs having a diameter of $2.1 \mathrm{~nm}$, which is 3 times smaller than that of red-emitting QDs, resulting in the decrease of negative charge of the surface of QDs, which is critical to generating the electric field between the nanoprobe and ITO bottom electrode. Since the amount of cargo delivered to a single cell is highly dependent on the concentrations of cargo assembled, sizes and surface charges of the cargo need to be considered for the electrical assembly and release in an efficient way. However, the aforementioned concerns cannot be a big issue because most of the materials of interest to be delivered to cells are larger than the size of quantum dots and also have enough charges on its surface (e.g., DNAs, RNAs, and proteins). ${ }^{30-32}$ Finally, multiple hNSCs were penetrated with the nanoprobe and subjected to the electrical force with the same parameters used for the delivery of QDs, proving that the technique used for the cargo delivery is not inducing the morphological changes of cells (Figure S8). Hence, this electrically controllable assembly and release technique is very powerful for the delivery of materials of interest into specific cells or the manipulation of single cells in a simple, rapid, easy to use, and reproducible manner. Moreover, since we used undifferentiated human NSCs that can be differentiated into neurons or glial cells when their differentiation ability is unlocked by the transfection of NeuroD gene, selective differentiation of NSCs is possible for the study of NSC differentiation at the single-cell level. ${ }^{33}$

\section{CONCLUSION}

Here, we introduced a new single-cell manipulation technology that is very powerful for the monitoring of cell penetration, the attachment of cargo on a nanoprobe, and its release into single hNSCs. By combining a new polymer-insulated nanoprobe with cell chip technology, all processes essential for single-cell manipulation were successfully conducted based on electrical methods. The polymer-insulated nanoprobe with a diameter of $250 \mathrm{~nm}$ was fabricated by sequential electrochemical and FIB etching, which is very sensitive to changes that occur in the environment around the nanoprobe. The spikelike currents that appeared on the $I-t$ graph were found to be very effective for sensitive monitoring of the cell penetration process. Moreover, the negatively charged QDs were assembled on the surface of the nanoprobe rapidly within less than $3 \mathrm{~min}$ via electrical force. The QDs attached on the surface of the nanoprobe were then released within $20 \mathrm{~s}$ in normal PBS solution when the reverse voltage was applied between the nanoprobe and the ITO bottom substrate. The thin layer of QDs generated on the surface of the nanoprobe was then successfully delivered into the nucleus of single human NSCs without using any chemical linkers or external stimuli. Since all the processes conducted in this study (the monitoring of cell penetration, the assembly of target materials, and the release of cargoes into a single cell) were based on electrical methods, the developed single-cell manipulation system can be applied for chip-based rapid singlecell manipulation systems that can deliver materials of interest (e.g., anticancer drugs, fluoresceins, siRNAs, and plasmid 

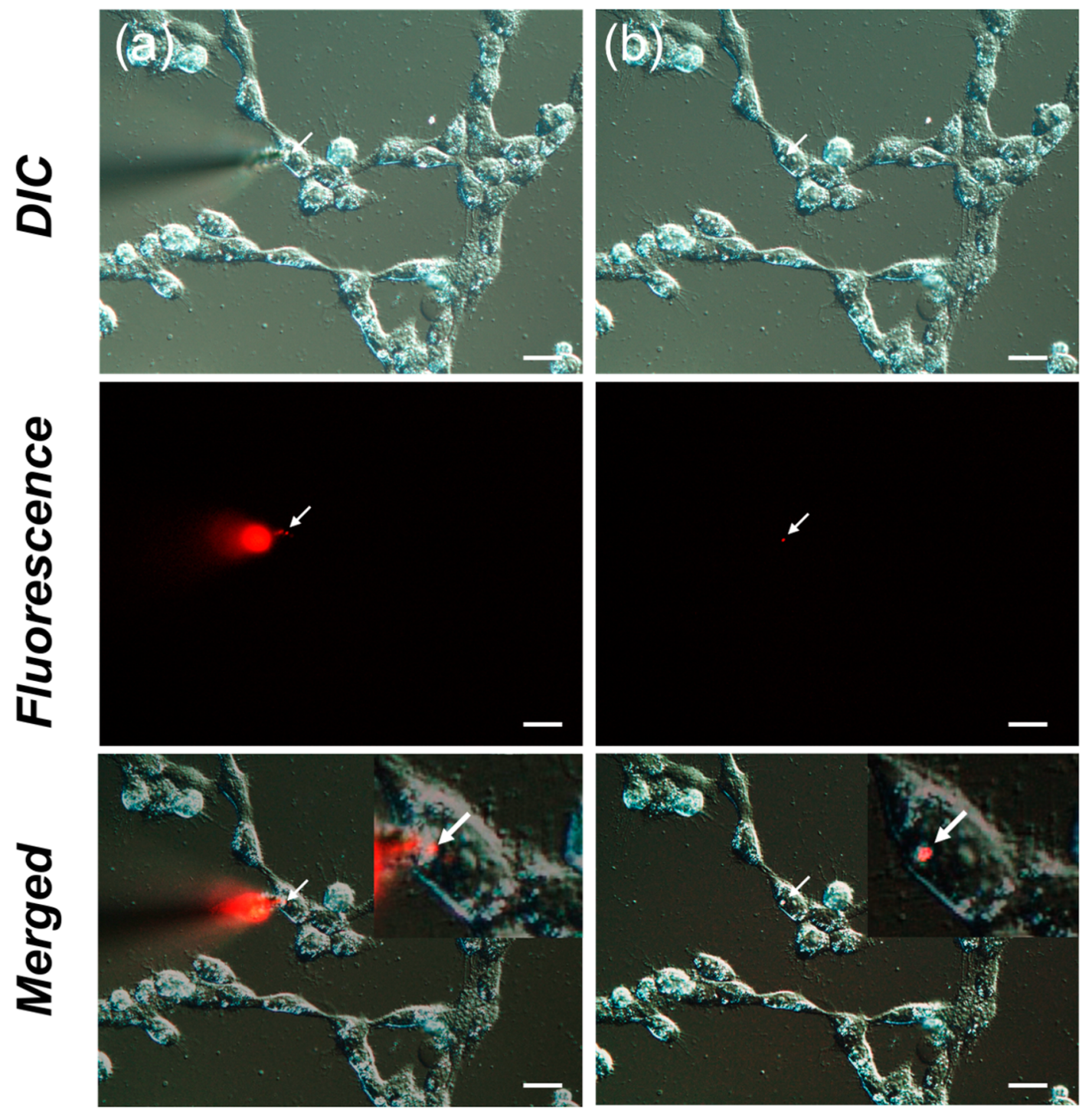

Figure 5. DIC, fluorescence, and merged images of hNSCs (a) during and (b) after the penetration for the electrical delivery of QDs into intracellular environment. White arrow in (a) and (b) indicates the exact spot of QDs delivered to single hNSC. Electrolyte used for the study was normal DMEM medium containing 10\% FBS and 1\% antibiotics. Images represented in (b) were taken after washing cells with DPBS and a normal culture medium. Scale bar $=25 \mu \mathrm{m}$.

vectors) into target cells when integrated with ectromechanical systems (MEMS) technology.

\section{ASSOCIATED CONTENT}

\section{S Supporting Information}

Whole setup for the electrical monitoring and delivery of cargo into single cells, gold nanoprobes fabricated by sequential electrochemical and FIB etching, polymer-insulated tungsten nanoprobe and its electrical characteristics when integrated with cell chip, cells images with high magnification for the precise control of penetration, reproducibility test of electrical assembly and release, thin layer of QDs assembled on the surface of a nanoprobe by electrical force, green-emitting QDs delivered to hNSCs, and the cell viability test for the electrically controlled delivery of QDs. This material is available free of charge via the Internet at http://pubs.acs.org.

\section{AUTHOR INFORMATION}

\section{Corresponding Author}

*Tel.: +82-2-705-8480. Fax: +82-2-3273-0331. E-mail: jwchoi@sogang.ac.kr.

\section{Notes}

The authors declare no competing financial interest.

\section{ACKNOWLEDGMENTS}

This work was supported by Samsung Research Funding Center of Samsung Electronics under Project (SRFC-MA140104). K.-B.L would like to acknowledge Perry T. Yin for his scientific input and valuable suggestions for the manuscript.

\section{ABBREVIATIONS}

hNSCs, human neural stem cells (HB1.F3)

QDs, quantum dots

FIB, focused ion beam

AFM, atomic force microscopy

$\mathrm{CNT}$, carbon nanotube 
SERS, surface-enhanced Raman spectroscopy ECM, extracellular matrix

ITO, indium tin oxide

DMEM, Dulbecco's modified eagle's medium

FBS, fetal bovine serum

\section{REFERENCES}

(1) Spiller, D. G.; Wood, C. D.; Rand, D. A.; White, M. R. H. Measurement of Single-Cell Dynamics. Nature 2010, 465, 736-745.

(2) Arakawa, T.; Noguchi, M.; Sumitomo, K.; Yamaguchi, Y.; Shoji, S. High-Throughput Single-Cell Manipulation System for a Large Number of Target Cells. Biomicrofluidics 2011, 5, 014114.

(3) Jager, E. W. H.; Inganas, O.; Lundstrom, I. Microrobots for Micrometer-Size Objects in Aqueous Media: Potential Tools for Single-Cell Manipulation. Science 2000, 288, 2335-2338.

(4) Kim, L. N.; Choi, S. E.; Kim, J.; Kim, H.; Kwon, S. Single Exposure Fabrication and Manipulation of 3D Hydrogel Cell Microcarriers. Lab Chip 2011, 11, 48-51.

(5) Lee, D.; Lin, B. J.; Lee, A. K. Hippocampal Place Fields Emerge upon Single-Cell Manipulation of Excitability During Behavior. Science 2012, 337, 849-853.

(6) Han, S. W.; Nakamura, C.; Obataya, I.; Nakamura, N.; Miyake, J. A Molecular Delivery System by Using AFM and Nanoneedle. Biosens. Bioelectron. 2005, 20, 2120-2125.

(7) Nakamura, C.; Kamiishi, H.; Nakamura, N.; Miyake, J. A Nanoneedle Can Be Inserted into a Living Cell without Any Mechanical Stress Inducing Calcium Ion Influx. Electrochemistry 2008, 76, 586-589.

(8) Obataya, I.; Nakamura, C.; Han, S.; Nakamura, N.; Miyake, J. Nanoscale Operation of a Living Cell Using an Atomic Force Microscope with a Nanoneedle. Nano Lett. 2005, 5, 27-30.

(9) Han, S. W.; Nakamura, C.; Imai, Y.; Nakamura, N.; Miyake, J. Monitoring of Hormonal Drug Effect in a Single Breast Cancer Cell Using an Estrogen Responsive GFP Reporter Vector Delivered by a Nanoneedle. Biosens. Bioelectron. 2009, 24, 1219-1222.

(10) Han, S. W.; Nakamura, C.; Kotobuki, N.; Obataya, I.; Ohgushi, H.; Nagamune, T.; Miyake, J. High-Efficiency DNA Injection into a Single Human Mesenchymal Stem Cell Using a Nanoneedle and Atomic Force Microscopy. Nanomedine 2008, 4, 215-225.

(11) Chen, X.; Kis, A.; Zettl, A.; Bertozzi, C. R. A Cell Nanoinjector Based on Carbon Nanotubes. Proc. Natl. Acad. Sci. U. S. A. 2007, 104, $8218-8222$

(12) Singhal, R.; Orynbayeva, Z.; Sundaram, R. V. K.; Niu, J. J.; Bhattacharyya, S.; Vitol, E. A.; Schrlau, M. G.; Papazoglou, E. S.; Friedman, G.; Gogotsi, Y. Multifunctional Carbon-Nanotube Cellular Endoscopes. Nat. Nanotechnol. 2011, 6, 57-64.

(13) Choi, J. W.; Bhusal, R.; Kim, T. H.; An, J. H.; Kim, H. Electrochemical Detection of Bisphenol A - Induced Neuronal Toxicity Using RGD Peptide Modified ITO Electrode Cell Chip. Mol. Cryst. Liq. Cryst. 2010, 519, 36-42.

(14) Kafi, M. A.; Kim, T. H.; An, J. H.; Choi, J. W. Electrochemical Cell-Based Chip for the Detection of Toxic Effects of Bisphenol-A on Neuroblastoma Cells. Biosens. Bioelectron. 2011, 26, 3371-3375.

(15) Kim, T. H.; El-Said, W. A.; Choi, J. W. Highly Sensitive

Electrochemical Detection of Potential Cytotoxicity of CdSe/ZnS Quantum Dots Using Neural Cell Chip. Biosens. Bioelectron. 2012, 32, 266-272.

(16) Yea, C. H.; Min, J.; Choi, J. W. The Fabrication of Cell Chips for Use as Bio-Sensors. BioChip J. 2007, 1, 219-227.

(17) Kim, T. H.; Lee, K. B.; Choi, J. W. 3D Graphene OxideEncapsulated Gold Nanoparticles to Detect Neural Stem Cell Differentiation. Biomaterials 2013, 34, 8660-8670.

(18) Kim, T. H.; El-Said, W. A.; An, J. H.; Choi, J. W. ITO/Gold Nanoparticle/RGD Peptide Composites to Enhance Electrochemical Signals and Proliferation of Human Neural Stem Cells. Nanomedicine 2013, 9, 336-344.

(19) Kim, S. U.; Nagai, A.; Nakagawa, E.; Choi, H. B.; Bang, J. H.; Lee, H. J.; Lee, M. A.; Lee, Y. B.; Park, I. H. Production and
Characterization of Immortal Human Neural Stem Cell Line with Multipotent Differentiation Property. Methods Mol. Biol. 2008, 438, 103-121.

(20) Lee, H. J.; Kim, K. S.; Kim, E. J.; Choi, H. B.; Lee, K. H.; Park, I. H.; Ko, Y.; Jeong, S. W.; Kim, S. U. Brain Transplantation of Immortalized Human Neural Stem Cells Promotes Functional Recovery in Mouse Intracerebral Hemorrhage Stroke Model. Stem Cells 2007, 25, 1204-1212.

(21) Strekal, N.; Kulakovich, O.; Belyaev, A.; Stsiapura, V.; Maskevich, S. Photoluminescence of Water-Soluble CdSe/ZnS Nanoparticles in Complexes with Cationic and Anionic Polyelectrolytes. Opt. Spectrosc. 2008, 104, 50-56.

(22) Cai, W. D.; Li, Y.; Dowding, R. J.; Mohamed, F. A.; Lavernia, E. J. A Review of Tungsten-Based Alloys as Kinetic Energy Penetrator Materials. Rev. Part. Mater. 1995, 3, 71-131.

(23) Lingwood, D.; Kaiser, H. J.; Levental, I.; Simons, K. Lipid Rafts as Functional Heterogeneity in Cell Membranes. Biochem. Soc. Trans. 2009, 37, 955-960.

(24) Simons, K.; Ikonen, E. Functional Rafts in Cell Membranes. Nature 1997, 387, 569-572.

(25) Skou, J. C. Enzymatic Basis for Active-Transport of Na+ and K+ Across Cell-Membrane. Physiol. Rev. 1965, 45, 596-618.

(26) Zhao, Z. Y.; Khisamutdinov, E.; Schwartz, C.; Guo, P. X. Mechanism of One-Way Traffic of Hexameric Phi29 DNA Packaging Motor with Four Electropositive Relaying Layers Facilitating Antiparallel Revolution. ACS Nano 2013, 7, 4082-4092.

(27) Wang, S. Y.; Haque, F.; Rychahou, P. G.; Evers, B. M.; Guo, P. X. Engineered Nanopore of Phi29 DNA-Packaging Motor for RealTime Detection of Single Colon Cancer Specific Antibody in Serum. ACS Nano 2013, 7, 9814-9822.

(28) Geng, J.; Wang, S. Y.; Fang, H. M.; Guo, P. X. Channel Size Conversion of Phi29 DNA-Packaging Nanomotor for Discrimination of Single- and Double-Stranded Nucleic Acids. ACS Nano 2013, 7, 3315-3323.

(29) Haque, F.; Lunn, J.; Fang, H. M.; Smithrud, D.; Guo, P. X. RealTime Sensing and Discrimination of Single Chemicals Using the Channel of Phi29 DNA Packaging Nanomotor. ACS Nano 2012, 6, 3251-3261.

(30) Sun, Q.; Liu, J.; Chen, M.; Miyake, J.; Qian, D. J. Fabrication and Characterization for the Nanoconjugates of PyridyldithioFunctionalized Multiwalled Carbon Nanotubes and Cytochrome $\mathrm{c}$ in Langmuir-Blodgett Films. J. Nanosci. Nanotechnol. 2014, 14, 54685472.

(31) Han, S. W.; Nakamura, C.; Miyake, J.; Chang, S. M.; Adachi, T. Single-Cell Manipulation and DNA Delivery Technology Using Atomic Force Microscopy and Nanoneedle. J. Nanosci. Nanotechnol. 2014, 14, 57-70.

(32) Mieda, S.; Amemiya, Y.; Kihara, T.; Okada, T.; Sato, T.; Fukazawa, K.; Ishihara, K.; Nakamura, N.; Miyake, J.; Nakamura, C. Mechanical Force-Based Probing of Intracellular Proteins from Living Cells Using Antibody-Immobilized Nanoneedles. Biosens. Bioelectron. 2012, 31, 323-329.

(33) Lee, H. J.; Kim, K. S.; Park, I. H.; Kim, S. U. Human Neural Stem Cells Over-Expressing VEGF Provide Neuroprotection, Angiogenesis and Functional Recovery in Mouse Stroke Model. PLoS One 2007, 2, e156. 